\title{
A THEORETICAL STUDY OF DESORPTION INDUCED BY ELECTRONIC TRANSITIONS IN ALKALI HALIDES
}

\author{
Noriaki ITOH *, A.M. STONEHAM and A.H. HARKER \\ Theoretical Physics Division, Harwell Laboratory, UKAEA, Oxon OXII ORA, UK
}

Received 5 December 1988; accepted for publication 3 March 1989

\begin{abstract}
We have studied desorption following the excitation of core- or valence-electrons using quantum chemical calculations of the potential energy surfaces. For core excitation, we simulate the state of two holes localised on a halogen ion using an extended semi-empirical molecular method (CNDO) to calculate the energy change associated with the displacement of the halogen ion or a neighbouring alkali ion. No instability was found for a bulk halogen ion with two localised holes, in agreement with recent experiments. However, also consistent with recent experiments, desorptive potential surfaces were obtained for both a $(100)$ surface halogen ion with two localised holes and for a (100) surface alkali ion when two holes are localised on the nearest subsurface (second layer) halogen ion. We use the results of our earlier CNDO calculations to evaluate the energetics of desorption induced by valence-electron excitation and indicate the possible origin of atomic desorption. The difference between the experimental features of the desorption induced by core- and by valence-electron excitation are explained in terms of the differences in the energy surfaces and hence the coupling of the electronic excited states with the lattice.
\end{abstract}

\section{Introduction}

Desorption induced by electronic transition (DIET) has been observed for some insulating solids and for a variety of adsorbate-metal systems $[1,2]$. Two different modes of electronic transition studied extensively are core-electron excitation and valence-electron excitation. In the former, excitation of a core electron is followed by an Auger transition which results in formation of a doubly-ionized anion, effectively two holes localised on an anion. According to Feibelman and Knotek [3], this hole localisation leads to desorption because of Coulomb repulsion between the doubly-ionized anion and neighbouring cations. On the other hand Menzel, Gomer and Redhead (MGR) [4] have suggested that electronic excitation yields an anti-bonding potential surface which leads to ejection of an atom from the surface.

* Permanent address: Department of Physics, Faculty of Science, Nagoya University, Furocho, Nagoya 464, Japan. 
Many theoretical studies have been carried out on electronically-induced desorption. For desorption induced by core-electron excitation, the main emphasis of theoretical studies has been placed on understanding the localisation of two holes over the time period, perhaps as long as $10^{-12} \mathrm{~s}$, required for atomic migration to take place $[5,6]$. It has been pointed out that the two-hole localisation has a sufficient lifetime if the hole-hole repulsion $U$ is greater than the width of the valence band. If $U$ is small compared with the bandwidth then [5] the two-hole state will spread rapidly throughout the solid into band-like states. Several calculations have been carried out too on the dissociation of molecules adsorbed on metal surfaces, and it has been shown that a potential surface which leads to desorption does indeed form upon two-hole localisation in covalent cases as well as ionic ones [7]. However, no detailed theoretical calculation for the desorption from condensed matter has been carried out. We shall show that quantum chemical methods can be extended to study this question.

Theoretical studies on desorption induced by valence-electron excitation are even more scanty. We shall exploit our earlier calculations for bulk processes modified, to include surface effects. Jennison and Emin [8] have suggested that small polaron-like self-trapping plays a role in the desorption of neutrals following valence-electron excitation, even though a specific calculation for silicon hydride does not conclusively predict an anti-bonding potential surface leading to desorption. Alternatively, Johnson and Inokuti [9] have suggested that desorption of $\mathrm{Ar}$ by valence-electron excitation is induced following recombination of an electron with a self-trapped hole (in this case, an $\mathrm{Ar}_{2}^{+}$ molecular ion) because of the large vibrational excitation of the molecular bond. The former case falls in the category known as "local excitation", and the latter example in that of "local heating" [10].

For adsorbate-metal systems, energy-resolved studies of neutral dissociation show that desorption may be induced by valence-electron excitation in some specific materials [7,11]. Desorption from silver and alkali halides [12], solid argon [13] and other condensed materials [14] have been reported.

Experimental work on desorption induced by core-excitation emphasises adsorbate-metal systems, which may be regarded as similar to two-dimensional insulators lying on metal surfaces. Condensed materials for which desorption induced by core excitation has been studied extensively are $\mathrm{SiO}_{2}$ [15] and alkali halides [16].

Amongst these, the alkali halides are the materials in which valence-electron excitation causes desorption, and hence it is convenient to compare desorption induced by core-electron excitation and valence-electron excitation in these materials. In the first place, valence-electron excitation of $\mathrm{NaCl}$ causes desorption dominated by halogen atoms [17], but $\mathrm{Cl}^{+}, \mathrm{Na}^{+}$and $\mathrm{Na}^{-}$are desorbed by core excitation of $\mathrm{NaCl}$ [16]. Secondly, desorbed particles are energetic for desorption by core-electron excitation $[16,17]$ but follow closely a 
Maxwellian for desorption by valence-electron excitation [18]. Thirdly, desorption of neutral alkali induced by valence-electron excitation exhibits a temperature dependence that is characteristic of thermal evaporation, while the temperature dependence of the desorption yield of excited alkali atoms is completely different $[16,19]$. Thus it is of interest to examine the consequences of valence-electron excitation and of core-electron excitation in alkali halides theoretically and to see how they are reflected in the experimental results.

The main purpose of the present paper is to investigate theoretically the electronic structure of the two-hole localised state and estimate the forces on the halogen ion with the two localised holes and on the surrounding ions. We can use essentially the same methods to study core excitation and to examine the atomic displacement that follows valence-electron excitation. We find that, following valence excitation, an anti-bonding potential surface is formed as a result of electron-lattice coupling, as suggested by Menzel, Gomer and Redhead.

\section{Desorption induced by core-electron excitation}

\subsection{Method of calculation: cluster calculations and the localising potential}

A CNDO semi-empirical molecular orbital approximation [20] was employed to obtain the wavefunctions and the energy of a $\mathrm{KCl}$ cluster in which a halogen ion with two localised holes was to be included. A basis of Slater orbitals consisting of $4 \mathrm{~s}$ and $4 \mathrm{p}$ orbitals of $\mathrm{K}$ and $3 \mathrm{~s}$ and $3 \mathrm{p}$ orbitals of $\mathrm{Cl}$ was used. In the CNDO method, the matrix elements of the Hamiltonian are approximated systematically, and are parameterised using the orbital exponent $\zeta$, ionisation energy $I$, electron affinity $A$ and bond parameter $\beta$. The values of the parameters determined previously [21] were used; these values gave an excellent description of the analogous (valence-excitation) photolysis process in the bulk of $\mathrm{KCl}$. It should be noted that the parameters used here were originally determined for the $\mathrm{KCl}$ molecule, and it was found that they could be carried over without change to the perfect solid. This parameter set, then, can treat adequately an even wider range of geometries and environments than are encountered in this surface study. The matrix calculation was carried out in a self-consistent way using the Harwell MOSES computer code [22].

The cluster consists of $27(3 \times 3 \times 3)$ ions, with either an alkali or halogen ion at the centre. For a cluster in the bulk, the bulk Madelung constant was used to take the effects of distant ions surrounding the cluster into account. Similarly the Madelung potential at the surface [23] was used for the cluster placed at the surface. The Madelung constants at distorted positions were calculated separately. By including the Madelung terms in this way, the 
sensitivity to unwanted surface effects (arising from finite cluster size) is reduced.

A conventional molecular orbital computation aims to obtain the state of a given symmetry which has the lowest energy. In general, this state is delocalised. When a hole is created rapidly correlation and dynamical effects [5] lead to a state in which the hole is localised on a particular ion. The surrounding ions will be polarized as a result. It would be possible to modify local orbital theories to treat this case, by maximizing the expectation value of a local operator in the manifold composed of the lowest virtual orbitals. A simpler method, which is more within the spirit of the CNDO scheme, is to apply a localising potential by shifting the ionisation potentials at one site. This shift must be large enough to ensure that the orbitals on that site do not mix with those on other sites and delocalise the hole. To obtain the wave function $\Phi$ of the cluster which included a halogen ion with two localised holes, we used as an intermediate step the Hamiltonian $H^{\prime}$ in which the ionisation potential of a halogen atom was reduced by an amount $\Delta I$ between 5 and $20 \mathrm{eV}$, and solved the wave equation

$H^{\prime} \Phi=\epsilon^{\prime} \Phi$,

where $\epsilon^{\prime}$ is the total energy and $\Phi$ the wave function of the cluster that includes the modified halogen ion. Using the same wave function, the total energy of the system which we need was obtained as the expectation value:

$\epsilon=\langle\Phi|H| \Phi\rangle$,

where $H$ is now the Hamiltonian in which the ionisation potential is not modified but $\Phi$ is the wavefunction from the modified Hamiltonian. In essence, we modify the cluster and allow all ions other than the modified one to polarise in response to the presence of the holes. We then use the current wavefunction and the unmodified Hamiltonian to give the total energy. We shall subtract the perfect cluster energy, calculated conventionally, so as to refer the total energy to that for delocalised holes.

The Mulliken charge $\rho$ on the halogen ion of which the ionisation potential is modified was obtained using

$\rho=e \sum_{i=1}^{4} \sum_{j=1}^{N} \nu_{j} C_{i j}^{2}$

where $e$ is the electron charge and $\nu_{j}$ the occupation number of the orbital $j$ and $C_{i j}$ the coefficient of the $j$ th one-electron function for the basis function $i$. Summation $i$ is made over the basis functions belonging to the halogen ion. We use values of $\rho$ to choose a suitable value of $\Delta I$, and keep the same value of $\Delta I$ in a range of calculations.

The value of change in $\rho$ (which we write as $\theta$ ) does not accurately represent the hole charge, for the following reason. Even with an unmodified 
ionisation potential the Mulliken charge on the halogen ions in the cluster is not uniform: the Mulliken charge of the central halogen ion is somewhat larger than that of the other 12 equivalent halogen ions, simply because our cluster is rather small. Thus by changing $\theta$, besides the hole localisation, there is a shift of the charge from the central halogen ion to the other ions. As $\Delta I$ increases, the value of $\theta$ saturates at 1.5 , instead of 2 . For most purposes, a satisfactory description is to scale the Mulliken charge. We shall then say the total hole charge is $q=4 \theta / 3$, which saturates at 2 . We shall specify when we use the Mulliken charge $\rho$, the increment $\theta$, or the scaled increment $q$.

In our calculations of core excitation, no displacement of ions surrounding the halogen ion with two localised holes has been taken into account. Indeed, CNDO calculations, in which the surrounding ions were displaced according to the results of a Mott-Littleton calculation using the Harwell HADES code, appeared to give total energies higher than without distortion. The distortion of the lattice around two localised holes suddenly generated appears to be much smaller than that predicted for the fully-relaxed geometries. In the following calculations on core excitation (though not of valence excitation) distortion of surrounding ions is not considered, apart from our specified ionic motion. Since the two localised holes are generated very rapidly (typically $10^{-16} \mathrm{~s}$, much smaller than the inverse of the lattice characteristic frequencies), we emphasise that our calculated results represent the potential energy imposed on the doubly ionised ion just after being generated.

\subsection{Localisation of two holes}

The wave equation was solved for a halogen-centred cluster for which the ionisation potential at the central atom was modified by various values of $\Delta I$. Examination of the wavefunctions and eigenvalues reveals that the lowest unoccupied orbital includes predominantly the basis functions belonging to the central halogen ion. The eigenvalue of this orbital $|k\rangle$ is found to be above the valence band. The energy difference between the eigenvalue of the orbital $|k\rangle$ and the top of the valence band, which is $9.6 \mathrm{eV}$ for $\Delta I=15 \mathrm{eV}$, arises from $\Delta I\left(\sum_{i} C_{i k}^{2}\right)$, where $C_{i k}$ are the coefficients in the expansion of orbital $|k\rangle$ in terms of the basis functions $|i\rangle$, and from the repulsion energy of two holes. Using the calculated wave functions, we find the repulsion energy of two holes on a single halogen to be $6.9 \mathrm{eV}$.

The relation between the total energy $\epsilon$ of the cluster and the Mulliken charge $\rho$ was calculated for several values of $\Delta I$ between 5 and $20 \mathrm{eV}$. We show in fig. 1 the relation between the change in total energy $E$ and the square of $\theta$ (the change in Mulliken charge on the ionised ion) as $\Delta I$ is increased from zero. The quadratic dependence of $E$ on $\theta$ involves two contributions, namely the repulsion of the two localised holes and the polarisation of the surroundings. The value of $E$ for $\Delta I=15 \mathrm{eV}$ is $6.8 \mathrm{eV}$, which agrees with the 


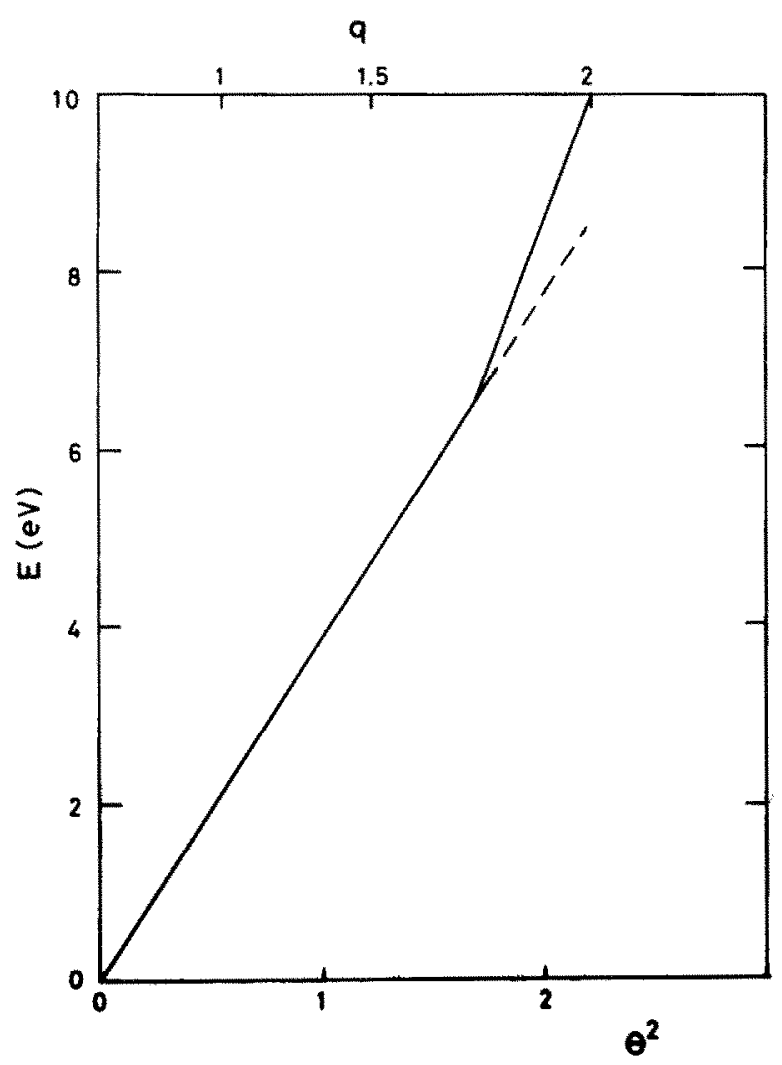

Fig. 1. The relation between the total cluster energy $E$ relative to the perfect cluster and the increment $\theta$ in the Mulliken charge density on the hole-localised halogen ion.

value obtained above. Deviation from the quadratic relation between $E$ and $\theta$ is seen for scaled charge $q \geq 2$, which occurs at about $\Delta I=15 \mathrm{eV}$. As $\Delta I$ increases further, the coefficient of the basis function for the hole-localised orbital in the lowest unoccupied orbital decreases, corresponding to delocalisation of the hole. This effect is associated with an improper choice of the ionisation potential as $\Delta I$ becomes larger. In what follows we use a value of $15 \mathrm{eV}$ for $\Delta I$.

The hole localisation energy, obtained from the quadratic relation and extrapolating to a hole charge of $q=2$, is $8.5 \mathrm{eV}$. The two-hole localisation energy for transition metal ions, obtained from the Auger electron spectrum, has nearly the same value [24]. We note that this hole localisation energy is substantially larger than the width (approximately $3 \mathrm{eV}$ ) of the valence band [25], so that in this case the condition for localisation [5,6] as a preliminary to desorption induced by core-electron excitation is satisfied. As noted above, 
there are two obvious components in the quadratic dependence of energy $E$ on the change $q$ in ionic charge. One part is intra-ionic, determined by the "absolute hardness" of the ion [26]. The ionic total energy under constant external potential is then $\eta q^{2}$. For atomic chlorine, values of $\eta$ of about $4.7 \mathrm{eV}$ are found both theoretically and experimentally. The second component is interatomic, namely the electronic polarisation of surrounding ions. The simplest estimate [27,28] gives a term $\left(q^{2} / 2 R\right)\left(1-\epsilon_{\infty}^{-1}\right)$, where $R$ is not normally the nearest neighbour distance; for a charge $q$ at a cation site with anions alone polarisable (approximately true here) $R$ is about 0.62 of the nearest neighbour distance $R_{0}$ [28]. We find an energy $(1.22 \mathrm{eV}) q^{2}$ with $R=R_{0}$ and $(1.94 \mathrm{eV}) q^{2}$ with $R=0.6 R_{0}$. Overall, one expects most of the quadratic change in energy to be intra-atomic in origin. The value from our calculations $E \approx(3.8 \mathrm{eV}) \theta^{2} \approx(2.2 \mathrm{eV}) q^{2}$ is smaller than one might have anticipated by these simple arguments.

\subsection{Is there a lattice instability for a doubly-ionised halogen ion in the bulk?}

Here we calculate the total energy $E$ of the cluster in which the central halogen atom is displaced along $\langle 100\rangle$ and $\langle 110\rangle$ directions. Fig. 2 shows the relation between $E$ and the displacement $\xi$, calculated with $\Delta I=15 \mathrm{eV}$. In this range of displacement, the displacement does not cause any change in the value of the Mulliken charge $\theta$ from 1.3. This means that the result shown in fig. 2 is the potential curve for the hole charge $q$ of 1.8 . If the value of $\Delta I=10$ $\mathrm{eV}$ had been used instead of $15 \mathrm{eV}$, the value of $\theta$ would depend strongly on $\xi$, implying incomplete localisation. Thus in the following calculations it is important for us to use $\Delta I=15 \mathrm{eV}$.

The results show clearly that energy increases with increasing displacement $\xi$, even though the change in the Madelung energy in this range of displacement is negligible. Thus the increase in the energy is ascribed to the Pauli repulsion between the halogen ion with two localised two holes (hereafter we describe it as $\mathrm{Cl}^{+}$) and neighbouring alkali ions, and not to long-range Coulomb interactions. In obtaining the result shown in fig. 2 , no allowance is made for displacements of the ion surrounding the $\mathrm{Cl}^{+}$ion, as noted above.

The results of the calculations shown in fig. 2 suggest that two-hole localisation in the bulk of alkali halides does not produce any instability that induces atomic displacement. This is in conflict with the suggestion by Varley, who argued [32] that an interstitial-vacancy pair is generated from two localised holes. In earlier experiments on the X-ray photon energy dependence of the F-centre yield by monochromatic X-rays, a step was found at the $\mathrm{ClK}$ edge in the yield of the $\mathrm{F}$ centres in $\mathrm{KCl}$ [29]. Such a step could also arise from the change in the distribution of secondary electrons upon crossing the inner shell electron energy, as has been demonstrated for $\mathrm{KBr}$ and $\mathrm{RbBr}$ [30]. In fact, recent experiments by Brown et al. [31] show no change in the F centre 


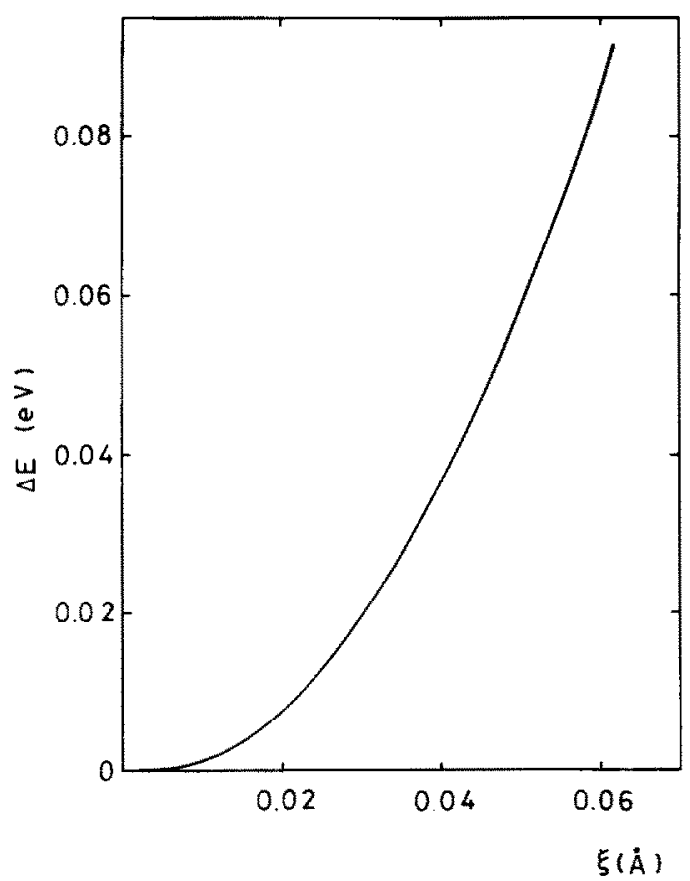

Fig. 2. Change $\Delta E$ in the total cluster energy induced by displacement of a $\mathrm{Cl}^{+}$ion along a $\langle 100\rangle$ direction.

yield upon crossing the $\mathrm{Cl} \mathrm{L}$ edge. Thus one can conclude, in agreement with our conclusion, that there is no experimental evidence for the Varley mechanism [32] at present. Even in the case that $F$ centres are created by inner-shell excitation, this may be associated with two self-trapping events located close to each other.

The two-hole localisation formed by an Auger transition within $10^{-15} \mathrm{~s}$ is very likely to be dissociated into two separate holes. Since the holes are very rapidly self-trapped and subsequently converted to self-trapped excitons after trapping electrons, two localised holes in the bulk might finally be converted to two self-trapped excitons close to each other. The consequence of forming a close pair of self-trapped excitons in terms of defect production is still an open question.

\subsection{Desorption induced by two-hole localisation}

We now turn to the possibility of core excitation yielding desorption. We have calculated the wavefunctions, eigenvalues and total energies of the clusters shown in fig. 3. For the configuration of fig. $3 \mathrm{a}$, holes initially were 

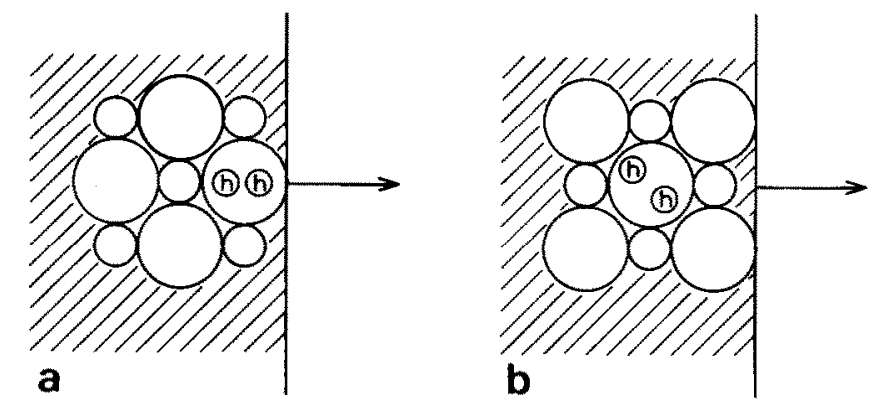

Fig. 3. The $\mathrm{KCl}$ clusters used in the calculation, showing the $\mathrm{K}^{+}$and $\mathrm{Cl}^{+}$displacements considered. The Madelung corrections for more distant ions were included too.

localised on the halogen ion at the centre of the top layer, while for the configuration of fig. $3 \mathrm{~b}$ the holes were localised on the halogen ion at the centre of the cluster when the ionisation potential was changed. The main features of the electronic structures are very similar to those of the clusters in the bulk. The value of $\theta$ for $\Delta I=15 \mathrm{eV}$ is 1.3 , as for the core-excited halogen ion in the cluster in the bulk. The Mulliken charge on the alkali ion is almost unchanged, indicating that the alkali ion is still largely unaffected. In each of the cases (figs. $3 a$ and $3 b$ ) the lowest unoccupied orbital is mainly composed of the $\mathrm{p}$ orbitals of the doubly-ionised halogen ion. These form a bonding-type interaction with neighbouring alkali ions. The absence of electrons in this orbital indicates that the Coulomb repulsion between the doubly-ionised halogen and neighbouring alkali ions is only weakly screened.

The Madelung energy as a function of displacement of an ion from the surface was calculated too. For the configuration of fig. 3a, the doubly-ionised halogen ion was displaced perpendicular to the surface, while for the configuration of fig. $3 b$ the alkali ion at the centre of the top layer was displaced. The relation between the Madelung energy and the displacements is shown in fig. 4. Evidently, localisation of two holes at the top layer of the surface causes a highly repulsive electrostatic potential for the halogen ion, and a similar localisation of two holes at a halogen in the second layer does the same for the nearest neighbour alkali ion at the top surface. Therefore we carried out a CNDO calculation for these two cases.

The relation between the total energy and the displacement $\xi$ of the doubly-ionised halogen ion for the configuration of fig. $3 \mathrm{a}$ is shown in fig. 5 . The Madelung energy is shown also for comparison. The value of the Mulliken charge $\theta$ on the halogen ion with two localised holes is practically unchanged for $\xi<0.15$, but decreases slightly for $\xi>0.15$ and becomes 1.2 at a displacement of 0.3 . The results shows that the doubly ionised halogen ion at the surface is certainly desorbed. The trend of the $E(\xi)$ relation is similar to the relation between the Madelung energy and $\xi$ for $\xi>0.05$, except that the slope 


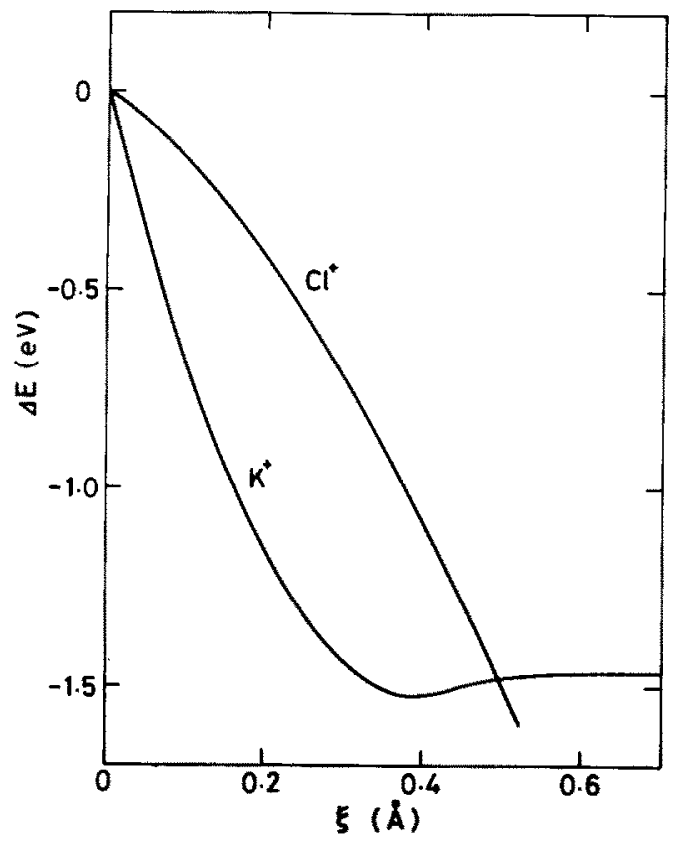

Fig. 4. Electrostatic potential for the displacement of $\mathrm{Cl}^{+}$and $\mathrm{K}^{+}$normal to the surface, corresponding to the motion shown in figs. $3 \mathrm{a}$ and $3 \mathrm{~b}$ respectively.

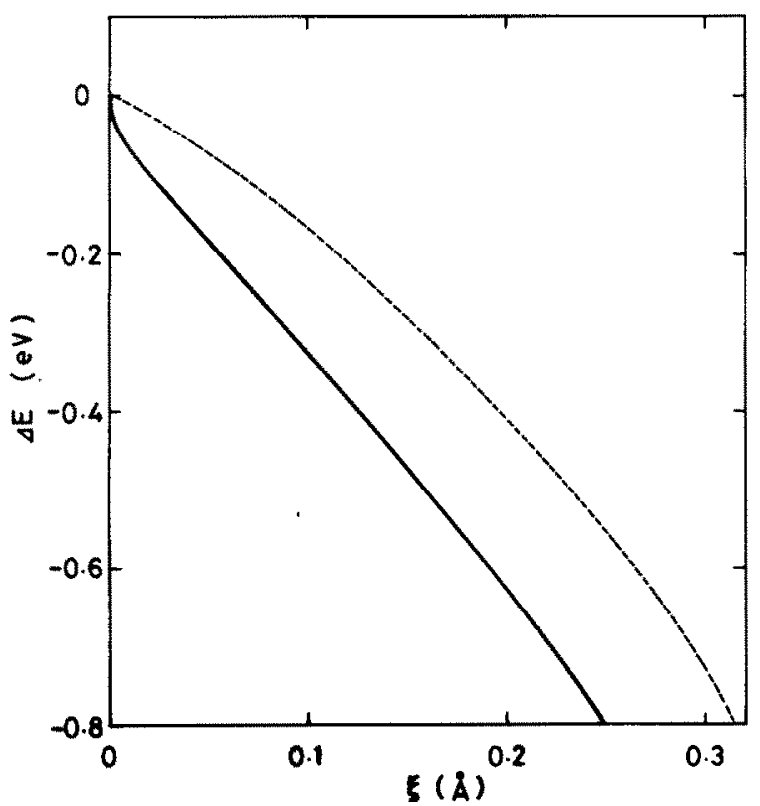

Fig. 5. Change $\Delta E$ in the total cluster energy induced by displacement of $\mathrm{Cl}^{+}$normal to the surface. The broken line shows the Madelung part, as in fig. 4 . 
of the $E(\xi)$ curve is slightly steeper. For $\xi<0.05$, the result of CNDO exhibits a short range repulsion, which may be ascribed to the Pauli repulsion.

A similar $E(\xi)$ relation was obtained for displacement of an alkali ion using the configuration of fig. $3 \mathrm{~b}$, and the result is shown in fig. 5 , where the $\xi$ dependence of the Madelung energy is also shown. The initial slope of the total energy calculated using CNDO is higher than that of the Madelung potential, as for the halogen shown in fig. 6 . In this case, however, the $E-\xi$ relation starts to level off as $\xi$ increases, even though there is no change in the Mulliken charge density. Further increases in $\xi$ cause an increase in $E$. This increase in $E$ is considered to be the effect of the finite size of the cluster. Since the potassium ion at the surface is strongly bound to the rest of the cluster of ions, its displacement modifies the wave function of the whole cluster and hence causes an indirect increase in the energy of the cluster. This charge would be distributed over the whole crystal in reality but, because of the finiteness of the cluster size, makes a noticeable contribution here to the total energy. In fact an increase of $\xi$ induces an overall increase in the eigenvalues. Thus we would expect the $E(\xi)$ relation in fig. 6 should be below the Madelung energy versus $\xi$ curve for a sufficiently large cluster.

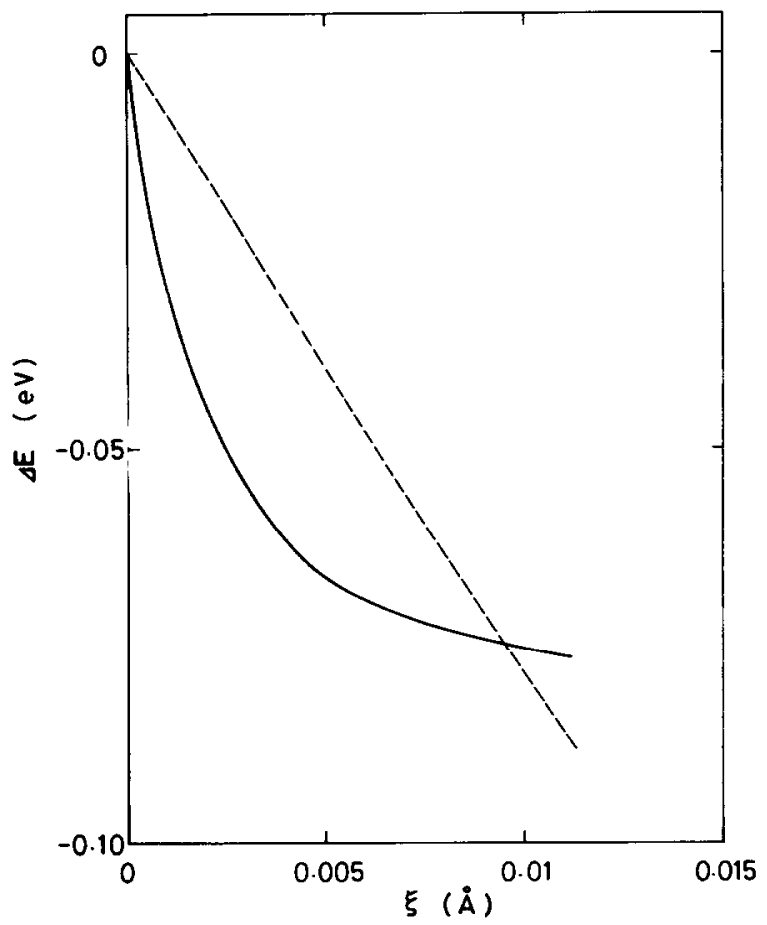

Fig. 6. Change $\Delta E$ in the total cluster energy induced by displacement of $\mathrm{K}^{+}$normal to the surface. The broken line shows the Madelung part, as in fig. 4. 


\subsection{Comparison with other approaches}

Walkup and Avouris [33] have used molecular dynamics to follow the transient motions associated with stimulated desorption in an approach making very different approximations. They consider a thin (4 or 6 layers) slab, and use Coulomb and short-range potentials. No electronic polarisation is included. It is not clear to us where the electrons removed in creating the core holes are placed, for the asymptotic behaviour is not what one would guess (see e.g. the 30 fs curve of their fig. 1, where the curve must surely be positive at large distances, but seems consistently negative). Possibly the $t=0$ positions were not in equilibrium but omitted surface rumpling (it is not certain their potentials give rumpling, but it seems likely) and there are some extra transients resulting. Whatever the explanation, it is clear that our calculation is complementary to theirs, since ours emphasises the chemical and electronic polarisation components which they omit.

\section{Desorption induced by valence-electron excitation}

In this section we shall exploit the parallels between the bulk processes of defect production after valence-electron excitation and the corresponding desorption processes for halogen atoms. The bulk processes have been studied extensively experimentally [14] and also theoretically [21] by the same class of semi-empirical methods used in section 2, though of course no localising potential is needed. For most of our present purposes, we shall need only to extend the conclusions for the bulk process by using energy-cycle arguments.

\subsection{Energy needed for desorption}

It has been well established that the valence-electron excitation of alkali halides leads to desorption of halogen atoms. The energy $E_{\mathrm{D}}$ needed to desorb a halogen atom can be evaluated using the Born-Haber cycle shown in table 1 ;

$E_{\mathrm{D}}=E_{\mathrm{H}}+A_{\mathrm{H}}-A_{\mathrm{F}}$,

where $E_{\mathrm{H}}$ is the formation energy of a halogen ion vacancy, $A_{\mathrm{H}}$ the electron affinity of a halogen atom and $A_{\mathrm{F}}$ the electron affinity of a halogen vacancy from the vacuum level, namely the sum of the thermal ionisation energy $I_{\mathrm{F}}$ of an $\mathrm{F}$ centre and the electron affinity $A_{\mathrm{C}}$ of the conduction band. Table 2 gives values of $E_{\mathrm{D}}$, calculated from eq. (4). These values are, of course, bulk values: $E_{\mathrm{H}}-A_{\mathrm{F}}$ can be somewhat different at surface sites. There are several terms which are identifiable (altered Madelung energies, surface dipoles arising from 
Table 1

The Born-Haber cycle for desorption of a halogen atom

$$
\begin{aligned}
\mathrm{Cl}^{-} \text {[in the bulk of a perfect crystal] } & \rightarrow\left[\text { bulk anion vacancy] }+\mathrm{Cl}_{\infty}^{-}-E_{\mathrm{H}}\right. \\
& \rightarrow\left[\text { bulk anion vacancy] }+\mathrm{Cl}_{\infty}^{0}+\mathrm{e}_{\infty}^{-}-E_{\mathrm{H}}-A_{\mathrm{H}}\right. \\
& \rightarrow\left[\text { bulk anion vacancy] }+\mathrm{e}_{\mathrm{c}}^{-}+\mathrm{Cl}_{\infty}^{0}-E_{\mathrm{H}}-A_{\mathrm{H}}+A_{\mathrm{c}}\right. \\
& \rightarrow\left[\text { bulk F centre] }+\mathrm{Cl}_{\infty}^{0}-E_{\mathrm{H}}-A_{\mathrm{H}}+A_{\mathrm{C}}+I_{\mathrm{F}}\right.
\end{aligned}
$$

Overall:

$\mathrm{Cl}^{-}$[in the bulk of a perfect crystal] $\rightarrow$ [bulk $\mathrm{F}$ centre $]+\mathrm{Cl}_{\infty}^{0}-E_{\mathrm{D}}$

$E_{\mathrm{D}}=E_{\mathrm{H}}+A_{\mathrm{H}}-A_{\mathrm{C}}-I_{\mathrm{F}}=E_{\mathrm{H}}+A_{\mathrm{H}}-A_{\mathrm{F}}$

$E_{\mathrm{D}}$ is the energy for desorption, $E_{\mathrm{H}}$ the energy of formation of a halogen ion vacancy, $A_{\mathrm{H}}$ the electron affinity of a vacancy from the vacuum level.

rumpling, image potential terms, etc.) though the broad picture is probably very similar.

\subsection{Energy available for desorption}

In table 2, we list also the band-gap energy and the energy of the free exciton. The energy possessed by a free exciton is certainly larger than $E_{\mathrm{D}}$, indicating that the emission of halogen from a free exciton is energetically possible. However, the free exciton in the bulk is converted very rapidly to a self-trapped exciton, and a self-trapped exciton has much less energy. There is thus a problem, since self-trapping and desorption of a halogen atom from the surface are competing processes.

\begin{tabular}{|c|c|c|c|c|c|c|c|c|c|}
\hline & \multirow[t]{2}{*}{$\begin{array}{l}\text { Band } \\
\text { gap }\end{array}$} & \multirow[t]{2}{*}{$\begin{array}{l}\text { Free } \\
\text { exciton }\end{array}$} & \multicolumn{2}{|c|}{$\begin{array}{l}\text { Self-trapped } \\
\text { exciton }\end{array}$} & \multicolumn{5}{|c|}{$\begin{array}{l}\text { Energy of desorption of a } \\
\text { halogen atom, } E_{\mathrm{D}}\end{array}$} \\
\hline & & & I & II & $\overline{E_{\mathrm{H}}}{ }^{\mathrm{g})}$ & $A_{\mathrm{H}}{ }^{\mathrm{h})}$ & $I_{\mathrm{F}}^{\mathrm{i}}$ & $A_{C}{ }^{j)}$ & $\begin{array}{l}E_{\mathrm{D}}= \\
E_{\mathrm{H}}+A_{\mathrm{H}} \\
A_{\mathrm{C}}-I_{\mathrm{F}}\end{array}$ \\
\hline $\mathrm{NaCl}$ & $8.8^{\text {a) }}$ & $7.96^{a)}$ & & & 5.12 & 3.61 & 1.94 & 0.5 & 6.3 \\
\hline $\mathrm{KCl}$ & $8.7^{\text {b) }}$ & $7.77^{b)}$ & $\begin{array}{l}4.5^{\mathrm{d})} \\
6.4^{\mathrm{e}} \\
7.1^{\mathrm{9}}\end{array}$ & $\begin{array}{l}3.4^{d)} \\
5.3^{\text {e) }} \\
6.0^{f)}\end{array}$ & 4.89 & 3.61 & 2.05 & 0.5 & 6.0 \\
\hline $\mathrm{KBr}$ & $7.5^{b)}$ & $6.79^{c)}$ & & & 4.60 & 3.36 & 1.80 & 0.8 & 5.4 \\
\hline
\end{tabular}

Table 2

Comparison of electronic excitation energy with energy of desorption of a halogen atom for alkali halides (in eV)

a) Ref. [45]. ${ }^{\text {b) }}$ Ref. [46]. ${ }^{\text {c) }}$ Ref. [47].

d) Energy in the lowest state.

e) Energy in the next electron-excited state.

f) Energy in the ionised self-trapped exciton.

g) Ref. [48]. ${ }^{\text {h) }}$ Ref. [49]. ${ }^{\text {i) }}$ Ref. [50]. ${ }^{\text {j) }}$ Ref. [25]. 
We estimated the energy of the lowest state of the self-trapped exciton by subtracting the self-trapping energy of a positive hole and the ionization energy of the self-trapped excitons from the band-gap energy. In this evaluation (referred to as I) the minima of the adiabatic potential surfaces for the ionized self-trapped exciton [34] and the self-trapped exciton at the lowest state were assumed to be at the same configuration. Note that the stabilization energy of a positive hole has been obtained theoretically to be $1.61 \mathrm{eV}$ (present work using the approach of refs. [36,37]; these use the energy difference of $\mathrm{Cl}^{0}$ and $\mathrm{Cl}_{2}^{-}$; ref. [35] gives $1.7 \mathrm{eV}$ ). Alternatively, the available energy of the self-trapped exciton can be written as the sum of the optical transition energy (i.e. for the Franck-Condon luminescence) to the ground state and the lattice distortion energy in the ground state, i.e. the energy needed to distort the perfect crystal to the self-trapped exciton geometry: this evaluation is referred so as II. We have estimated the energy to give two halogens their distortion for the self-trapped configuration in the ground state to be $1.07 \mathrm{eV}$.

Table 2 shows values obtained for the lowest energy of the self-trapped excitons, of its next highest electron-excited state and of its ionized state, using methods I and II. There are modest but not trivial differences seen between the values obtained using the two different methods. This discrepancy may arise from the assumption that the ionic configuration of the self-trapped hole and of the self-trapped excitons are the same. Whether this is because of the off-centre nature of the self-trapped exciton $[38,39]$ or because of the state-dependent screening of the hole charge by the electron, we presume that the ground state distortion energy is less accurate for the self-trapped exciton. Thus we concentrate on the energies obtained using method I for further discussion.

\subsection{Comparison with other situations}

Even if we take the values derived by method $I$, the lowest state of the self-trapped exciton does not have sufficient energy to cause desorption. Accordingly, only an excited self-trapped cxciton can lead to desorption of halogen atoms. It has been established that defect formation in the bulk of alkali halides is induced from excited states of the self-trapped exciton, not from the lowest state of the self-trapped exciton [40]. The conversion from an excited state of the self-trapped exciton to a pair consisting of an $F$ centre and an $\mathbf{H}$ centre is considered to occur following an anti-bonding adiabatic potential surface which connects these two states, in agreement with ref. [21]. Thus is it likely that there exists a similar adiabatic potential surface from an excited state of the self-trapped exciton leading to desorption of a halogen atom. Despite the limitations of accuracy, the energy cycles combine with explicit calculations to confirm that the anti-bonding potential surface suggested by Menzel, Gomer and Redhead in alkali halides does exist, but only as 
a result of the significant electron-lattice coupling. This description applies to the desorption of halogen atoms. Loubriel et al. [41] describe the desorption of alkali from specimens enriched with $F$ centres.

The energy cycle approach also has application to desorption of positrons from alkali halides, where again it appears that excited excitons are important. After the implantation of energetic positrons into ionic crystals, positrons are seen to be reemitted with a maximum kinetic energy close to the band gap [42]. The sequence of events proposed involves positronium $\left(\mathrm{e}^{+} \mathrm{e}^{-}\right)$formation, positronium thermal diffusion, and interaction with some species $S$ at the surface such that $\mathbf{S}$ removes the electron and the positron is emitted. The original suggestion was that $S$ was a valence band hole at the bottom of the valence band. Unfortunately, the timescale for hole relaxation rules this out: positron emission occurs after hundreds of picoseconds, whereas the hole will lose energy rapidly by coupling mainly to longitudinal optic phonons, rising to the top of the valence band and self-trapping in a few picoseconds. In such cases the maximum energy available is $I_{\mathrm{S}}-I_{\mathrm{P}}-I_{\mathrm{A}}$, with $I_{\mathrm{S}}$ the energy for capture of a conduction electron by surface species $\mathrm{S}, I_{\mathrm{P}}$ the (small) positronium ionisation energy, and $I_{\mathrm{A}}$ the (small) positron affinity in the crystal. Putting in values, one finds:

(1) The main part of the observed emitted positron energy spectrum can be understood if unrelaxed excitons are involved.

(2) There is still a high-energy tail which needs further explanation.

(3) With self-trapped excitons in their ground states, neither component can be understood without some extra energy contribution.

\section{Conclusion}

We have compared new calculations for energy surfaces following core excitation with those deduced previously for valence excitation. We would emphasise the remarkable differences between the potential surfaces leading to the desorption in these two cases. There may well be further differences arising from gas-phase processes [43] which we do not discuss but which will surely affect the distribution of charge states seen. Different surfaces, particularly on small particles, may also give different results. After core excitation the Auger transition creates a two-hole localised state before any lattice relaxation is induced. A strong repulsive potential for either alkali or halogen ions, arising from both Coulombic and Pauli repulsions, will induce instantaneous emission of energetic ions, notably $\mathrm{Cl}^{+}$and $\mathrm{Na}^{+}$. On the other hand, valence-electron excitation near the surface leads to self-trapped excitons in highly excited states, which are de-excited emitting phonons. Desorption is induced if the self-trapped configuration possesses an excited state from which an adiabatic potential surface leads to emission of a halogen atom $\mathrm{Cl}^{0}$. In this case the 
desorption is a slow process requiring at least $10^{-12} \mathrm{~s}$. Since the adiabatic potential surface is not a normal mode of the system, the energy of vibrational motion in this reaction coordinate for the excited state is dissipated quite rapidly to a variety of phonon modes (see e.g. Masri and Stoneham [44]). Thus the desorbed atoms will have energies only slightly larger than the thermal energy. The loss of neutral $\mathrm{Cl}^{0}$ will leave a surface excess of alkali which, it is usually assumed, can be evaporated. Thus the main features of desorption following each excitation mechanism can be understood in a common quantum-chemical approach.

\section{Acknowledgement}

The work described in this report is part of the longer term research carried out within the Underlying Programme of the UKAEA.

\section{References}

[1] N.H. Tolk, M.M. Traum, J.C. Tully and T.E. Madey, Eds., Desorption Induced by Electronic Transitions I (Springer, Berlin, 1983).

[2] W. Brenig and D. Menzel, Eds., Desorption Induced by Electronic Transitions II (Springer, Berlin, 1985).

[3] M.L. Knotek and P.J. Feibelman, Phys. Rev. Letters 40 (1978) 964;

P.J. Feibelman and M.L. Knotek, Phys. Rev. B 18 (1978) 6531.

[4] D. Menzel and R. Gomer, J. Chem. Phys. 41 (1964) 3311;

P. Readhead, Can. J. Phys. 42 (1964) 886.

[5] M. Cini, Solid State Commun. 20 (1976) 605; Phys. Rev, B 17 (1978) 2788.

[6] G.A. Sawatzky, Phys. Rev. Letters 39 (1977) 504.

[7] D.E. Ramaber, in; ref. [2], p. 10, and references therein.

[8] D.R. Jennison and D. Emin, Phys. Rev. I.tters 51 (1983) 1390.

[9] R.E. Johnson and M. Inokuti, Nucl. Instr. Methods 206 (1982) 289.

[10] A.M. Stoneham, Rept. Progr. Phys. 44 (1981) 1251.

[11] P. Feuler, in: ref. [2], p. 142.

[12] H. Kanzaki and T. Mori, Semicond. Insulators 5 (1983) 401; Phys. Rev. B 29 (1984) 3573.

[13] J. Schou, P. Borgesen, O. Ellegard and H. Sorensen, in: ref. [2], p. 170, and references therein.

[14] N. Itoh, Nucl. Instr. Methods 132 (1976) 281; and to be published.

[15] M.L. Knotek, and J.E. Houston, J. Vacuum Sci. Technol. 20 (1982) 544; B 1 (1983) 899.

[16] R.F. Haglund, Jr., R.G. Albridge, D.W. Cherry, R.K. Cole, M.H. Mendenhall, W.C.B. Peatman, N.H. Tolk, D. Niles, G. Margaritondo, N.G. Stoffel and E. Taglauer, Nucl. Instr. Methods B13 (1985) 525.

[17] N.H. Tolk, W.E. Collins, J.S. Kraus, R.J. Morris, T.R. Pian, N.G. Stoffel and G. Margaritondo, in: ref. [1], p. 156.

[18] H. Overeijinder, M. Szymonski, A. Haring and A.E. de Vries, Radiation Effects 38 (1978) 21; 36 (1978) 63.

[19] E. Taglauer, N. Tolk, R. Riedel, E. Colavita, G. Margaritondo, N. Gershenfeld, N. Stoffel, J.A. Kelber, G. Loubriel, A.S. Bommanavar, M. Bakshi and Z. Huric, Surface Sci. 169 (1986) 267. 
[20] J.A. Pople and D.L. Beveridge, Approximate Molecular Orbital Theory (McGraw-Hill, New York, 1970).

[21] N. Itoh, A.M. Stoneham and A.H. Harker, J. Phys. C 10 (1977) 4197.

[22] A.H. Harker and S.B. Lyon, AERE-R.8598 (1979).

[23] J. Magill, J. Bloem and R.W. Ohse, J. Chem. Phys. 76 (1982) 6228.

[24] E. Antonides, E.C. Jase and G.A. Sawatzky, Phys. Rev. 15 (1977) 1669.

[25] R.T. Poole, J.G. Jenkin, J. Liesegang and R.C. Leckey, Phys. Rev. B 11 (1975) 5179.

[26] K.D. Sen, P.C. Schmidt and M.C. Bohm, J. Phys. B 18 (1985) L35.

[27] N.F. Mott and R.W. Gurney, Electronic Processes in Ionic Crystals (Oxford Univ. Press, Oxford, 1948).

[28] A.M. Stoneham, Electronic Processes in Ionic Semiconductors, AERE Report M3523 (1985).

[29] J. Sharma and R. Smoluchowski, Phys. Rev. 137 (1965) A259.

[30] N. Itoh, J. Sharma and R. Smoluchowski, Abstract for International Symposium on Color Centres in Alkali Halides, Urbana, 1965, p. 78.

[31] F.C. Brown, B.R. Sever and J.P. Stott, Phys. Rev. Letters 57 (1986) 2279.

[32] J.H.O. Varley, Nature 174 (1954) 886.

[33] R.E. Walkup and Ph. Avouris, Phys. Rev. Letters 56 (1986) 524.

[34] R.T. Williams and M.N. Kabler, Phys. Rev. B 9 (1974) 1897.

[35] A.N. Jette, T.L. Gilbert and T.P. Das, Phys. Rev. 184 (1969) 884.

[36] P.E. Cade, A.M. Stoneham and P.W. Tasker, Phys. Rev. B 30 (1984) 4621.

[37] P.E. Cade, A.M. Stoneham and P.W. Tasker, Phys. Rev. B 33 (1986) 4166.

[38] C.H. Leung, G. Brunet and K.S. Song, J. Phys. C 18 (1985) 4459;

see also: R.T. Williams, K.S. Song, W.L. Faust and C.H. Leung, Phys. Rev. B 33 (1986) 7232.

[39] D. Block, A. Wasiela and Y. Merle d'Aubigne, J. Phys. C 11 (1978) 4201.

[40] N. Itoh, Advan. Phys. 32 (1982) 491;

see also: K. Tanimura and N. Itoh, J. Phys. Chem. Solids 45 (1984) 323.

[41] G.M. Loubriel, T.A. Green, P.M. Richards, R.G. Albridge, D.W. Cherry, R.K. Cole, R.F. Haglund, Jr., L.T. Hudson, M.H. Mendenhall, D.M. Newns, R.T. Savundaraj, K.J. Snowden and N.H. Tolk, Phys. Rev. Letters 57 (1986) 1781.

[42] A.P. Mills, Jr. and W.S. Crane, Phys. Rev. Letters 53 (1984) 2165.

[43] R.E. Walkup, Ph. Avouris and A.P. Ghosh, Phys. Rev. B 36 (1987) 4577.

[44] P.M. Masri and A.M. Stoneham, J. Electron. Mater. 14 (1984) 205.

[45] T. Miyata and T. Tomiki, J. Phys. Soc. Japan 24 (1968) 1286.

[46] T. Tomiki, J. Phys. Soc. Japan 23 (1967) 1280.

[47] T. Tomiki, T. Miyata and H. Tukamoto, J. Phys. Soc. Japan 35 (1973) 495.

[48] K. Diller, AERE-TP.642 (1975).

[49] R.C. Weast, Ed., Handbook of Chemistry and Physics (CRC Press, Cleveland, 1986).

[50] J.J. Markham, F Centers in Alkali Halides (Academic Press, New York, 1966) p. 123. 